\title{
DESEMPENHO DA PRODUÇÃO INDUSTRIAL NOS ESTADOS BRASILEIROS: UMA ANÁLISE DA HIPÓTESE DA DESINDUSTRIALIZAÇÃO SUBNACIONAL ${ }^{1}$
}

\author{
Paulo Vítor Veloso Almeida \\ Graduando em Ciências Econômicas pelo Instituto de Economia da Universidade Federal de \\ Uberlândia \\ Email: paulovveloso@hotmail.com \\ Michele Polline Veríssimo \\ Professora do Instituto de Economia da Universidade Federal de Uberlândia \\ Email: micheleverissimo@ufu.br
}

\begin{abstract}
RESUMO: Este trabalho analisa o desempenho da indústria de transformação dos estados brasileiros, com o objetivo de verificar a ocorrência de desindustrialização subnacional. Para isso, foram analisados indicadores da atividade industrial dos estados no período 1996-2013, tais como Valor da Transformação Industrial (VTI), Densidade Produtiva, População Ocupada e Composição das Exportações. Os dados sugerem que especialmente os estados do Sul e Sudeste vêm perdendo participação dos indicadores da atividade industrial de transformação no período analisado. Portanto, conclui-se que o processo de desindustrialização ocorre de forma desigual nos estados brasileiros.
\end{abstract}

PALAVRAS-CHAVE: Indústria de Transformação; Desindustrialização; Estados Brasileiros.

CLASSIFICAÇÃO JEL: L16, L60, O14.

ABSTRACT: This paper analyzes the Brazilian states manufacturing industry performance in order to verify the occurrence of subnational deindustrialization. For this, it analyzes the industrial indicators of the states in the period 1996-2013, such as Industrial Manufacturing Value (VTI), Productive Density, Employment Rate and Exports Composition. The data suggests that especially in South and Southeast regions the manufacturing industrial indicators have loosing share in the analyzed period. Therefore, it is possible to conclude that there is an inequality of deindustrialization process in the Brazilian states.

KEY-WORDS: Manufacturing Industry; Deindustrialization; Brazilian States.

Artigo submetido para o Encontro Nacional de Economia Industrial e Inovação (ENEI)

\footnotetext{
${ }^{1}$ Os autores agradecem o CNPq pelo apoio financeiro na pesquisa
} 


\section{INTRODUÇÃO}

O processo de industrialização da economia brasileira passou por diversos momentos ao longo do tempo, dentre os quais se destacam as décadas de 1950 a 1970, onde o processo industrial avançou significativamente. Após esse período, a industrialização brasileira sofreu um desaquecimento nos anos 1980 e 1990, em virtude de políticas econômicas aplicadas especialmente com a instauração do Plano Real: abertura comercial e financeira, elevadas taxas de juros, ajustes fiscais (corte de gastos do governo, privatizações e aumento da carga tributária) e taxa de câmbio sobrevalorizado. Tais medidas foram aplicadas com a finalidade de conter o processo inflacionário, porém contribuíram para o desaquecimento da produção industrial, a quebra de empresas e o aumento do desemprego.

Além das políticas econômicas supracitadas, ao longo dos anos 2000, a economia brasileira passou por um período de expressiva produção e exportação de produtos intensivos em recursos naturais, em um contexto onde as commodities estavam valorizadas no mercado internacional e com uma demanda externa que favorecia tais produtos. Neste período, estes bens alcançaram maior participação no saldo comercial, em virtude dos grandes volumes de exportação. Por outro lado, a indústria de transformação perdeu participação nas exportações e no Produto Interno Bruto (PIB) nacional.

Estas mudanças suscitaram uma discussão acerca da "reprimarização" da pauta de exportação brasileira, em virtude do atual cenário que favorecia a exportação de produtos primários, além das vantagens comparativas inerentes aos mesmos (abundância de recursos naturais e mão de obra), o que, por um lado, aumenta o volume de exportação, mas, por outro lado, como tais produtos apresentam baixo valor agregado, esse evento acarreta menores efeitos multiplicadores de renda e emprego sobre a economia quando comparados aos produtos mais sofisticados e de maior valor agregado (BRUNO ET AL., 2009).

O processo de apreciação cambial vigente neste período continuou por fazer com que as exportações de produtos de baixo valor agregado continuassem a crescer, apesar da valorização cambial proporcionar uma importação de insumos mais baratos, o que possivelmente facilitaria o crescimento da participação da indústria. Todavia, considerando que o processo de transformação industrial exige um grande volume de capital e mão de obra qualificada, o câmbio desfavorável desestimulou a produção e a exportação de manufaturas, que necessitam de um câmbio depreciado para elevar a participação da indústria na balança comercial (BRESSER-PEREIRA; MARCONI, 2008).

Tendo em vista este contexto, esta pesquisa pretende investigar a ocorrência de um processo de desindustrialização, com base na análise do perfil industrial das diferentes regiões e Unidades Federativas do Brasil. O que justifica a análise subnacional é que, apesar de a economia brasileira possivelmente passar por alterações em seu padrão industrial, é visível a disparidade dessa dinâmica sobre as diferentes regiões/estados do país, os quais possuem matrizes industriais características que são afetadas de modo diferente pelos condicionantes econômicos. Com isso, objetiva-se demonstrar os diferentes perfis de produção e exportação dos estados brasileiros, afim de obter indicativos de uma possível perda de relevância da atividade industrial para os resultados econômicos nacionais e regionais.

\section{FUNDAMENTAÇÃO TEÓRICA}

\subsection{Conceitos de desindustrialização}

De acordo com Kaldor (1966), a indústria é um fator de grande importância em uma economia, pois esta seria geradora de emprego e produto, possibilitando o processo de crescimento econômico. Seguindo a argumentação de Kaldor (1966), os ganhos obtidos pelas 
exportações de commodities deveriam ser aproveitados para investir no crescimento dos setores mais avançados tecnologicamente, no sentido de retomar o processo de substituição de importações e permitir que o alívio à restrição externa ocorra sobre bases estáveis. Isso implica investimento nos setores industriais de média e alta tecnologia, possibilitando assim que uma economia se industrializasse com os rendimentos das exportações de commodities.

Segundo Rowthorn e Wells (1987), a desindustrialização seria apenas o resultado natural de um processo de desenvolvimento econômico. Este processo poderia decorrer, no caso dos países centrais, da maior elasticidade-renda do setor de serviços em relação à indústria, ou mesmo da competição de produtos importados dos países menos desenvolvidos. Sendo assim, o conceito natural consiste basicamente na perda de participação da indústria na geração do produto quando o setor de serviços avança. Tal processo pode não ser negativo, quando se trata do país ou região ter alcançado certo nível de desenvolvimento econômico e naturalmente migre para o setor de serviços.

Rowthorn e Ramaswany (1999) apud Oreiro e Feijó (2010) definiram o chamado conceito “clássico" de desindustrialização, que se explica por uma redução persistente da participação do emprego industrial no emprego total de um país ou região. Analisando este conceito, os chamados países desenvolvidos ou do "primeiro mundo" teriam passado por um forte processo de desindustrialização a partir da década de 1970. A América Latina teria passado pelo mesmo processo na década de 1990, o que coincide com o período de implantação das políticas liberalizantes associadas ao "Consenso de Washington”. Tregenna (2009) apud Oreiro e Feijó (2010) redefiniu o conceito de desindustrialização de forma mais ampla, como sendo uma situação na qual tanto o emprego industrial como o valor adicionado da indústria se reduzem como proporção do emprego total e do PIB, respectivamente.

Neste contexto, Carvalho e Kupfer (2007) comparam a evolução da matriz industrial brasileira com outros países selecionados, e concluem que, no Brasil, ocorre um processo de reversão da indústria, o qual para os países desenvolvidos ocorreu em um nível de renda superior. Com isso, ele demonstra a naturalidade o processo de desindustrialização ao mesmo tempo em que conclui que o mesmo ocorreu de forma precoce no Brasil.

Dentro do processo de desindustrialização, alguns autores, tais como Bresser-Pereira e Marconi (2008), trabalham com o conceito conhecido como Doença Holandesa. Este tipo de desindustrialização decorre da existência abundante de recursos naturais que geram vantagens comparativas ao país, levando-o a se especializar na produção de bens primários, e não se industrializar ou terminar se desindustrializando, o que desaceleraria o processo de desenvolvimento e crescimento econômico.

Segundo Bresser-Pereira e Marconi (2008), o processo de desindustrialização no Brasil foi motivado pela ruptura de políticas econômicas da década de 1990 que detinham seus efeitos. Por outro lado, o aumento do preço das commodities observada a partir de 2003 motivada pelo crescimento da demanda global, principalmente chinesa, permitiu que os exportadores brasileiros desses produtos se consolidassem no comércio mundial mesmo diante de uma taxa de câmbio real apreciada, ou no mínimo, acima do que os autores denominam de "taxa de câmbio de equilíbrio industrial", que seria aquela necessária para o setor de comercializáveis que integram tecnologia ser rentável à exportação.

Outros autores ressaltam a influência das políticas econômicas como fatores relevantes para o processo de desindustrialização. Neste sentido, Palma (2005) argumenta que a mudança do regime de substituição de importações por políticas de liberalização na década de 1990, aliado à política de abertura comercial e financeira, teria provocado um retrocesso da estrutura produtiva em função de uma especialização em produtos primários nos quais os países teriam vantagens comparativas ricardianas.

Utilizando a mesma linha de argumentação teórica, Cano (2012) destaca que, no caso do Brasil, o processo de “desindustrialização precoce e nociva” teria como causas principais a 
política de câmbio valorizado, a abertura descontrolada, a elevada taxa de juros, a redução do investimento direto externo (IDE) e a desaceleração da economia mundial. Neste contexto, o autor conclui sobre a forte tendência de reprimarização da pauta exportadora, na qual a participação dos produtos manufaturados no volume total de exportação foi reduzida, enquanto as importações de bens de consumo duráveis aumentaram, deslocando a demanda por manufaturas para o exterior e desestimulando o aquecimento da indústria de transformação brasileira.

\subsection{Desindustrialização subnacional}

No que se refere à discussão sobre o processo de desindustrialização subnacional, existe uma menor quantidade de literatura específica, porém nota-se um avanço de estudos sobre o tema.

Considerando trabalhos que agregam todos os estados, Botelho, Sousa e Avellar (2014), partindo de indicadores de valor adicionado, emprego, produtividade, densidade industrial e inserção externa, concluem que a incidência desigual do processo de desindustrialização nos estados e grandes regiões brasileiras não foi suficiente para alterar de forma significativa a forte concentração da indústria no Centro-Sul do país. Pois, ao se verificar, por exemplo, a intensidade da queda da relação VTI (Valor da Transformação Industrial)/PIB nas regiões Sul e Sudeste, em detrimento do aumento da mesma nas demais regiões, não se verifica uma migração intensa da atividade industrial para os outros estados.

Monteiro e Lima (2014) mostra uma tendência de reconfiguração do valor adicionado nacional da indústria de transformação entre as regiões brasileiras, com o Sudeste perdendo participação ao longo dos últimos 25 anos, o Sul se destacando nos ganhos, e as demais regiões elevando levemente suas respectivas participações. Outro resultado encontrado foi o baixo nível de renda per capita do Nordeste, relativamente ao Sudeste e Sul, no momento em que a indústria de transformação começou a perder participação, levando a crer que nesta região o processo possui um perfil precoce.

Em pesquisa sobre a região do Nordeste do Brasil, Libânio (2012) conclui que o crescimento econômico dos estados nordestinos no período 1996-2008 foi influenciado de forma positiva pela participação de manufaturas de média e alta tecnologia do total exportado e pelo complemento em relação à pauta de exportações da China.

Diversos autores se dedicam à análise do padrão industrial a partir de estudos de casos (estados) individuais. Por exemplo, para o estado de São Paulo, o estudo de Ferreira (2003) observa que a perda de participação do emprego industrial em relação ao setor de serviços se justifica mais pelo aumento do setor informal e pela precarização do trabalho, do que propriamente por uma substituição de empregos industriais pelos de serviços. Segundo este autor, não se pode falar em desindustrialização no estado de São Paulo, dado que houve um movimento de desconcentração industrial da capital para o interior ou mesmo para outros estados.

Rezende e Santos (2007) apontam que as fábricas do ABC paulista migraram para outras regiões do país e do próprio estado em busca de salários menores e de incentivos fiscais. As empresas que permaneceram na região intensificaram seus investimentos em tecnologia, ampliando a produtividade e provocando desemprego industrial local, sendo que tal rearranjo produtivo regional foi catalisado pelas políticas macroeconômicas das décadas de 1990 e 2000.

Arroyo (2012) apresenta que a pauta exportadora do estado de São Paulo é constituída basicamente por produtos industrializados, que respondem, desde 1997, por mais de $90 \%$ do total estadual. Assim, a autora conclui que se trata de um estado no qual a participação da 
indústria no PIB total é relativamente alta, traduzindo-o como um exemplo claro de uma região industrializada, que pode ser observado pelos índices de VTI e VBPI.

Analisando o estado do Rio de Janeiro, Rosendo e Britto (2011) mostram que os setores de refino e de exploração e produção de petróleo e gás lideraram o crescimento industrial do estado na primeira metade dos anos 2000. No entanto, apesar da forte expansão do VTI fluminense, puxado pelas atividades petrolíferas, a indústria do Rio de Janeiro continuou em processo de deterioração, mostrando que seu declínio cíclico permanece.

Teixeira e Rodolfo (2012) apontam que as políticas de fomento à indústria adotadas pelo governo federal e estadual não foram suficientes para conter a redução relativa do peso da indústria em Santa Catarina. Seguindo a mesma linha teórica, Cavalieri et al. (2013) argumentam que as políticas macro e microeconômicas pouco atrativas ao investimento produtivo produziram uma perda de importância relativa da indústria catarinense, expressa pela diminuição da produção e emprego industrial no produto e emprego total da economia estadual.

No que se refere ao perfil da economia do estado do Espírito Santo, Medeiros e Lannes Junior (2014) nos mostra que a participação da indústria no PIB é superior à média nacional. Essa é uma situação que poderia ser até encarada como algo positivo, não fosse o fato de que a participação das atividades da indústria de transformação com capacidade de gerar maior valor agregado ser muito inferior ao observado no restante do país.

Almeida e Sousa (2014) apresenta que, entre 1960 e 2010, a indústria mineira aumentou a sua participação relativa no VTI nacional em 94,9\%. Somente o Paraná apresentou um ganho de participação relativa maior do que Minas Gerais, com aumento de $117,6 \%$ no mesmo período. No caso de Minas Gerais, as vantagens comparativas do agronegócio estadual contribuíram para o desenvolvimento da indústria química, com a expansão do segmento de defensivos agrícolas e fertilizantes. O entrave ao crescimento da indústria mineira, para que o estado possa almejar ganhos de participação ainda mais acentuados no VTI nacional, está relacionado ao segmento de fabricação de materiais elétricos, eletrônicos e aparelhos de comunicação.

Barbosa et al (2015) analisam que no estado do Paraná, no período de 1996 a 2012, ainda que as evidências sinalizem para uma desindustrialização, ao analisar as diferentes microrregiões do estado, não se comprova isto. Pelo contrário, uma boa parte das microrregiões tiveram positivas taxas anuais de crescimento tanto da participação do emprego quanto da participação do valor adicionado da indústria. Para além disto, especialmente para os municípios com menor desenvolvimento industrial, não se tem nenhum vestígio da presença de um processo de desindustrialização.

A partir dessa revisão inicial da literatura, pretende-se analisar os dados referentes à participação da indústria de transformação dos estados brasileiros a fim de observar a possibilidade de vigência de um processo de desindustrialização em curso no país. Para isso, a próxima seção analisa os principais indicadores de desempenho industrial segmentados conforme as cinco regiões geográficas do Brasil.

\section{RESULTADOS E DISCUSSÃO}

A fim de realizar uma análise comparativa da evolução da atividade industrial nos estados brasileiros, o primeiro indicador a ser analisado é o da participação do VTI da indústria de transformação no VTI da indústria geral estadual. Esta variável será tratada como uma proxy do valor adicionado pela indústria de transformação em cada estado. Sendo assim, aqueles estados que apresentarem maiores porcentagens são aqueles no qual a indústria de transformação é predominante em detrimento da atividade extrativa. 
Tabela 1: Participação do VTI da indústria de transformação, Unidades da Federação, 1996-2013 (em \%)

\begin{tabular}{|c|c|c|c|c|c|c|}
\hline & 1996 & 2000 & 2005 & 2010 & 2013 & $\Delta(2013-1996)$ \\
\hline BRASIL & 96,59 & 94,49 & 91,42 & 88,26 & 86,32 & $-10,27$ \\
\hline MG & 90,19 & 87,68 & 83,17 & 75,48 & 69,69 & $-20,50$ \\
\hline $\mathbf{R J}$ & 89,98 & 78,76 & 68,19 & 67,49 & 62,49 & $-27,49$ \\
\hline SP & 99,55 & 99,65 & 99,75 & 99,35 & 98,36 & $-1,19$ \\
\hline ES & 99,18 & 99,36 & 99,38 & 99,52 & 99,40 & 0,22 \\
\hline PR & 98,54 & 97,85 & 98,56 & 98,56 & 97,11 & $-1,43$ \\
\hline SC & 99,13 & 99,46 & 99,31 & 99,27 & 99,22 & 0,09 \\
\hline RS & 79,91 & 77,89 & 61,15 & 45,44 & 44,07 & $-35,84$ \\
\hline MS & 93,5 & 93,44 & 94,55 & 90,51 & 91,16 & $-2,34$ \\
\hline MT & 98,86 & 98,76 & 99,15 & 99,12 & 98,48 & $-0,38$ \\
\hline GO & 94,09 & 92,4 & 95,6 & 93,08 & 94,18 & 0,09 \\
\hline DF & 99,22 & 99,28 & 99,6 & 98,67 & 99,16 & $-0,06$ \\
\hline RO & 95,92 & 97,08 & 96,85 & 97,03 & 96,21 & 0,29 \\
\hline AC & n.d. & 100 & 99,67 & 99,37 & 99,58 & $-0,42^{*}$ \\
\hline AM & 98,27 & 96,26 & 91,43 & 94,14 & 95,48 & $-2,79$ \\
\hline $\mathbf{R R}$ & 100 & n.d. & 99,18 & 96,15 & 90,68 & $-9,32$ \\
\hline PA & 67,32 & 60,38 & 54,01 & 21,77 & 19,11 & $-48,21$ \\
\hline AP & 51,6 & n.d. & 95,29 & 22,65 & 56,15 & 4,55 \\
\hline TO & 95,01 & 88,87 & 91,47 & 89,33 & 91,25 & $-3,76$ \\
\hline MA & 99,02 & 98,69 & 74,6 & 63,55 & 94,13 & $-4,89$ \\
\hline PI & 99,37 & 98,8 & 98,41 & 97,09 & 95,69 & $-3,68$ \\
\hline CE & 99,4 & 99,29 & 99,26 & 98,56 & 98,49 & $-0,91$ \\
\hline $\mathbf{R N}$ & 54,94 & 45,56 & 41,26 & 58,98 & 58,2 & 3,26 \\
\hline PB & 95,19 & 94,67 & 94,79 & 94,84 & 95,25 & 0,06 \\
\hline $\mathbf{P E}$ & 98,79 & 98,76 & 99,46 & 97,91 & 89,86 & $-8,93$ \\
\hline AL & 99,55 & 99,47 & 98,64 & 98,52 & 98,36 & $-1,19$ \\
\hline SE & 75,9 & 62,5 & 53,18 & 55,83 & 58,75 & $-17,15$ \\
\hline BA & 93,73 & 93,76 & 93,36 & 93,41 & 89,61 & $-4,12$ \\
\hline
\end{tabular}

Fonte: Elaboração própria a partir dos dados da PIA-IBGE (2016)

n.d.: dado não disponível

* variação entre 2000 e 2013

A análise da evolução do VTI, apresentada na Tabela 1, mostra que, em relação ao Brasil, o que se tem é uma redução de 10,27 p.p na participação do VTI da indústria de transformação em relação ao VTI total da indústria no período 1996-2013. Este fato mostra que no âmbito nacional existe uma perda de participação da indústria de transformação frente à indústria extrativa na geração de valor para a atividade industrial.

No geral, apenas sete dos 27 estados tiveram um aumento relativo da participação da indústria de transformação, sendo que apenas o Amapá e o Rio Grande do Norte apresentaram aumento superior a 1 p.p. Os outros 20 estados contaram com uma redução da participação da indústria de transformação no VTI, com destaque para os estados do Pará (-48,21 p.p.), Rio Grande do Sul (-35,84 p.p.), Rio de Janeiro (-27,5 p.p.) e Minas Gerais (-20,5 p.p.), que tiveram as maiores reduções de participação da atividade industrial de transformação perante ao avanço da atividade extrativa.

Na Região Sudeste, apenas um estado apresentou aumento de participação do VTI da indústria de transformação no período analisado, porém de pouca magnitude, Espírito Santo (0,22 p.p.). Os demais estados da região perderam capacidade da indústria de transformação de gerar valor. Cabe ressaltar o caso do Rio de Janeiro, as atividades relacionadas à exploração de recursos naturais começaram a ganhar foco, principalmente com a descoberta do petróleo do Pré-Sal em 2006. 
Na Região Sul, a situação é bem parecida com a do Sudeste, onde dois estados reduziram a participação do VTI da indústria de transformação, que são eles o Rio Grande do Sul (-35,84 p.p.), seguido por Paraná (-1,43 p.p.). Por outro lado, o estado de Santa Catarina apresentou um aumento praticamente insignificante de 0,09 p.p. Este fato sugere que, assim como no Sudeste, na região Sul, a perda de participação da indústria de transformação é significativa.

No Centro Oeste, três estados apresentaram redução da participação do VTI da indústria de transformação, porém de pequena magnitude: Mato Grosso (-2,34 p.p.), Mato Grosso do Sul (-0,38 p.p.), e Distrito Federal (-0,06 p.p.). Apenas o estado de Goiás contou com aumento relativo de 0,09 p.p, no período. A região Centro Oeste também é exemplo do aumento da capacidade de geração de valor das atividades relacionadas aos recursos naturais.

Por fim, nas regiões Norte e Nordeste, 12 dos 16 estados reduziram participação no VTI da indústria de transformação no total do estado, destacando Pará (-48,21 p.p.), Sergipe (-17,15 p.p.), Roraima (-9,32 p.p.) e Pernambuco (-8,93 p.p.). No Amapá (4,55 p.p.), Rio Grande do Norte (3,26 p.p.), Roraima $(0,29$ p.p.) e Pernambuco $(0,06)$, verificou-se aumento relativo de participação do VTI industrial de transformação.

Portanto, verifica-se que o indicador VTI da indústria de transformação tem perdido participação relativa na geração de valor industrial, embora o processo não seja homogêneo entre as diversas Unidades da Federação brasileira. Todavia, as perdas sobrepujam os ganhos, indicando, de forma preliminar, deterioração da atividade de transformação no país.

A Tabela 2 representa a participação no VTI da indústria de transformação no PIB de cada estado brasileiro. Este índice mostra o quanto a indústria de transformação é capaz de agregar de valor ao PIB do estado, ou seja, quanto maior o valor do índice, maior vai ser a participação da indústria de transformação na geração de produto/renda total.

Tabela 2: Participação do VTI no PIB estadual, Unidades da Federação, 1996-2013 (\%)

\begin{tabular}{ccccccc}
\hline & 1996 & 2000 & 2005 & 2010 & 2012 & $\Delta(2012-1996)$ \\
\hline MG & 17,64 & 21,12 & 23,11 & 20,89 & 19,43 & 1,80 \\
RJ & 13,20 & 13,60 & 14,82 & 14,18 & 13,33 & 0,13 \\
SP & 25,63 & 26,76 & 27,86 & 23,75 & 23,87 & $-1,76$ \\
ES & 10,51 & 17,11 & 15,96 & 11,25 & 9,95 & $-0,56$ \\
PR & 17,29 & 21,38 & 25,44 & 25,85 & 26,01 & 8,72 \\
SC & 23,70 & 25,33 & 25,89 & 25,21 & 25,90 & 2,20 \\
RS & 20,71 & 25,47 & 24,23 & 22,09 & 24,57 & 3,86 \\
MS & 7,12 & 6,92 & 12,35 & 14,23 & 16,88 & 9,77 \\
MT & 9,04 & 8,77 & 16,07 & 15,70 & 17,53 & 8,49 \\
GO & 9,55 & 9,80 & 15,16 & 17,41 & 17,99 & 8,44 \\
DF & 0,90 & 1,38 & 1,47 & 1,40 & 1,41 & 0,51 \\
RO & 2,76 & 4,04 & 8,08 & 7,78 & 7,01 & 4,25 \\
AC & $n . d$ & 1,50 & 2,05 & 2,95 & $n . d$ & $1,45 * *$ \\
AM & 42,19 & 46,46 & 54,08 & 46,54 & 44,35 & 2,16 \\
RR & 0,33 & $n . d$ & 1,07 & 0,97 & 0,92 & 0,59 \\
PA & 8,14 & 10,41 & 11,31 & 6,70 & 6,53 & $-1,61$ \\
AP & 2,76 & $n . d$ & 5,67 & 1,46 & $n . d$ & $-1,30$ \\
TO & 1,66 & 2,29 & 2,90 & 3,49 & 4,02 & 2,35 \\
MA & 6,06 & 7,37 & 6,49 & 4,01 & 6,19 & 0,13 \\
PI & 4,27 & 3,28 & 6,18 & 6,85 & 5,12 & 0,85 \\
CE & 10,61 & 14,64 & 13,02 & 13,36 & 12,93 & 2,32 \\
RN & 6,34 & 7,74 & 6,75 & 9,55 & 9,80 & 3,46 \\
PB & 8,63 & 8,98 & 10,38 & 9,85 & 10,10 & 1,47 \\
PE & 12,57 & 10,75 & 10,86 & 12,68 & 13,11 & 0,54 \\
AL & 19,82 & 17,39 & 14,89 & 11,44 & 12,80 & $-7,02$ \\
& & & & & &
\end{tabular}




$\begin{array}{lllllll}\text { SE } & 5,97 & 9,81 & 10,45 & 8,07 & 8,19 & 2,22\end{array}$

$\begin{array}{lllllll}\text { BA } & 12,32 & 20,83 & 24,61 & 20,91 & 20,74 & 8,42\end{array}$

Fonte: Elaboração própria a partir dos dados da PIA-IBGE (2016)

n.d.: dado não disponível

* variação entre 1996 e 2010

** variação entre 2000 e 2010

Ao analisar a participação do VTI da indústria de transformação no PIB de cada estado, verifica-se uma tendência de queda da participação da indústria na geração de produto em dois estados da Região Sudeste, especialmente em São Paulo, que configura o maior polo industrial do país. Em contraposição, alguns estados ganharam participação da atividade industrial no produto, como é o caso dos estados de Minas Gerais e dos estados sulinos: Paraná, Santa Catarina e Rio Grande do Sul, evidenciando que nesses últimos estados os maiores aumentos observados para o valor da transformação industrial entre 1996 a 2012.

Nas regiões, Nordeste e Centro-Oeste, observa-se que a maior parte dos estados, 17 dentre os 20, obtiveram variação positiva, sendo que em apenas 5 estados, a variação percentual foi inferior a 1 p.p, que é o caso do Distrito Federal (0,51 p.p), Roraima (0,59 p.p), Maranhão (0,13 p.p), Piauí (0,85 p.p) e Pernambuco (0,54 p.p). No mais, temos que apenas, $15 \%$ dos estados dessas regiões apresentaram uma redução da participação do VTI em relação ao PIB estadual, que são, Pará (-1,61 p.p), Amapá (-1,30 p.p) e Alagoas (-7,02 p.p).

Deste modo, quando se analisa a participação do VTI da indústria de transformação em relação ao PIB estadual é observado que, no período de 1996 a 2012, a maioria dos estados ganharam participação da indústria no PIB. Entretanto, ao analisar o período 2000 a 2010, tem-se que os valores são, na maioria, bem superiores aos encontrados em 2012, o que mostra que, apesar de a variação 1996 a 2012 ser positiva, o valor de 2012 representa uma queda em relação aos anos anteriores.

A Tabela 3, exibida na sequência, ilustra a evolução da participação do VTI da indústria de transformação dos estados em relação ao dado para o Brasil. Este índice permite fazer uma análise mais complexa da possibilidade da existência do processo de desindustrialização subnacional, pois esclarece que a maioria dos estados, nos quais se concentram os maiores valores da transformação industrial, estão passando por um processo de redução desta participação no VTI total do país. Se observado uma redução da participação do VTI do estado no Brasil, esta pode ser considerada uma evidência preliminar de desindustrialização. Porém, a análise deve ser mais apurada, pois um aumento da participação de alguns estados pode não estar compensando a redução de outros estados de maior relevância, e, em âmbito nacional, o país pode sofrendo o processo de desindustrialização.

Os dados revelam que quatro estados apresentaram uma diminuição relativa de sua participação na indústria de transformação brasileira - Rio de Janeiro, São Paulo, Pará e Alagoas. Ressalta-se que São Paulo, embora ainda seja o estado com maior peso na produção industrial do país (média de 44\% no período), teve a queda mais representativa (-11,77 p.p.). Por outro lado, 23 estados aumentaram a participação do VTI da indústria de transformação no total do Brasil, contudo, apenas quatro estados - Paraná, Goiás, Mato Grosso e Santa Catarina, tiveram aumento superior a 1 p.p.

Tabela 3: Participação do VTI da indústria de transformação dos estados no VTI Brasil, 1996-2013 (em \%) 


\begin{tabular}{ccccccc}
\hline & 1996 & 2000 & 2005 & 2010 & 2013 & $\Delta(2013-1996)$ \\
\hline MG & 8,44 & 8,84 & 9,59 & 10,10 & 9,44 & 1,00 \\
RJ & 8,06 & 7,91 & 7,89 & 7,95 & 7,62 & $-0,44$ \\
SP & 50,90 & 47,22 & 43,65 & 40,78 & 39,14 & $-11,77$ \\
ES & 1,09 & 1,65 & 1,62 & 1,27 & 1,36 & 0,28 \\
PR & 5,37 & 6,15 & 6,94 & 7,73 & 8,40 & 3,03 \\
SC & 4,57 & 4,56 & 4,76 & 5,29 & 5,70 & 1,13 \\
RS & 7,85 & 8,67 & 7,53 & 7,68 & 8,28 & 0,42 \\
MS & 0,35 & 0,33 & 0,58 & 0,85 & 1,20 & 0,84 \\
MT & 0,50 & 0,54 & 1,30 & 1,29 & 1,63 & 1,13 \\
GO & 1,09 & 1,07 & 1,65 & 2,34 & 2,73 & 1,64 \\
DF & 0,22 & 0,27 & 0,25 & 0,29 & 0,28 & 0,06 \\
RO & 0,07 & 0,10 & 0,22 & 0,25 & 0,30 & 0,23 \\
AC & n.d. & 0,01 & 0,02 & 0,03 & 0,04 & $0,03 *$ \\
AM & 3,40 & 3,24 & 3,89 & 3,83 & 3,51 & 0,11 \\
RR & 0,00 & n.d. & 0,01 & 0,01 & 0,01 & 0,01 \\
PA & 0,70 & 0,83 & 0,95 & 0,72 & 0,63 & $-0,07$ \\
AP & 0,03 & $n . d$. & 0,05 & 0,02 & 0,05 & 0,02 \\
TO & 0,02 & 0,04 & 0,06 & 0,08 & 0,12 & 0,09 \\
MA & 0,33 & 0,37 & 0,35 & 0,25 & 0,40 & 0,06 \\
PI & 0,12 & 0,08 & 0,15 & 0,21 & 0,17 & 0,05 \\
CE & 1,17 & 1,38 & 1,15 & 1,43 & 1,53 & 0,35 \\
RN & 0,26 & 0,29 & 0,26 & 0,42 & 0,46 & 0,20 \\
PB & 0,36 & 0,35 & 0,38 & 0,43 & 0,48 & 0,13 \\
PE & 1,62 & 1,21 & 1,17 & 1,66 & 1,81 & 0,19 \\
AL & 0,69 & 0,56 & 0,45 & 0,39 & 0,40 & $-0,28$ \\
SE & 0,18 & 0,27 & 0,30 & 0,27 & 0,30 & 0,12 \\
BA & 2,58 & 4,03 & 4,82 & 4,44 & 4,02 & 1,45 \\
\hline FE E & 0,30 & &
\end{tabular}

Fonte: Elaboração própria a partir dos dados da PIA-IBGE (2016)

n.d.: dado não disponível

* variação entre 2000 e 2013

A Região Sudeste reúne os estados com maior relevância na produção industrial do país, sendo que, no período, média de $62 \%$ dos resultados industriais brasileiros foram gerados pela indústria de transformação desta região. Todavia, o Sudeste vem perdendo participação relativa da atividade de transformação na economia brasileira, de 68,5\% em 1996 para 57,5\% em 2013. Dois estados reduziram sua participação, São Paulo (-11,77 p.p.) e Rio de Janeiro (-0,44 p.p.), enquanto Minas Gerais (1 p.p.) e Espírito Santo (0,28 p.p.) tiveram avanços poucos expressivos. Isso significa que a região que concentra a grande parte do parque industrial do país vem passando por um processo de redução do seu peso na atividade industrial, com desconcentração industrial para outros estados.

A Região Sul concentra cerca de $20 \%$ da atividade industrial de transformação do país no período, com aumento de participação de 18\% em 1996 para 22,3\% em 2013. Todos os estados desta região apresentaram um crescimento relativo da participação do VTI no total do Brasil, sendo que o destaque fica para o estado do Paraná (3,03 p.p.) seguido por Santa Catarina (1,13 p.p.) e Rio Grande do Sul (0,42 p.p.).

A Região Nordeste constitui o terceiro parque industrial do país, com peso médio de 8,8\% na produção industrial do país no período, e também contou com aumento de participação do VTI da indústria de transformação, de 7,3\% em 1996 para 9,6\% em 2013. Nesta região, destaca-se o estado da Bahia, que ganhou 1,45 p.p. de participação no VTI da indústria de transformação brasileira, em decorrência, principalmente, da instalação de parque automobilístico no estado (fábrica da Ford em Camaçari). 
A Região Norte manteve participação média no VTI brasileiro de 4,66\% no período. O destaque da região é o estado do Amazonas, que tem a maior participação no VTI nacional e avançou 0,11 p.p., muito em função da Zona Franca de Manaus.

Por último, a Região Centro Oeste, que detém a menor participação média do VTI da indústria de transformação no total brasileiro (3,75\%), também aumentou esta participação de 2,1\% em 1996 para 5,8\% em 2000. Todos os estados apresentaram um aumento de participação na geração de valor dentro do país, sendo que o destaque fica para Goiás $(1,64$ p.p.), seguido de Mato Grosso (1,13 p.p.).

Assim, a análise dos dados da Tabela 3 sugere que existe uma desconcentração da atividade industrial no país, em que houve deslocamento de empresas dos estados da Região Sudeste para outras Unidades da Federação. Contudo, cabe ressaltar que o aumento da participação dos estados nos resultados industriais do país em uma condição em que vários estados têm perdido relevância das respectivas indústrias de transformação pode estar mais associado à própria queda do VTI do país como um todo do que ao aumento do VTI estadual.

A Tabela 4 apresenta o índice de densidade produtiva industrial, obtida pela razão entre o VTI e o VBPI. Este índice visa avaliar a evolução da capacidade das atividades industriais em agregar valor na produção nacional. De acordo com Almeida, Feijó e Carvalho (2007, p.1) apud Botelho et al. (2014), quando o indicador é decrescente, há um maior uso de insumos importados representando, para o conjunto da indústria, transferência de produção e de seu respectivo valor agregado para o exterior. Ou seja, quanto maior o índice, mais a produção é intensiva em valor agregado gerado no próprio país.

Tabela 4: Densidade produtiva (VTI/VBPI), Unidades da Federação, 1996-2013 (em \%)

\begin{tabular}{ccccccc}
\hline & 1996 & 2000 & 2005 & 2010 & 2013 & $\Delta 2013-1996$ \\
\hline BRASIL & $\mathbf{4 6 , 7 1}$ & $\mathbf{4 4 , 2 9}$ & $\mathbf{4 1 , 2 3}$ & $\mathbf{4 3 , 2 4}$ & $\mathbf{4 2 , 0 1}$ & $\mathbf{- 4 , 7 0}$ \\
MG & 42,18 & 42,01 & 39,99 & 42,12 & 40,81 & $-1,37$ \\
RJ & 54,54 & 54,02 & 52,21 & 51,81 & 47,60 & $-6,94$ \\
SP & 48,24 & 44,98 & 42,09 & 43,54 & 42,56 & $-5,68$ \\
ES & 48,84 & 54,71 & 49,42 & 40,25 & 42,13 & $-6,72$ \\
PR & 42,89 & 40,77 & 38,36 & 43,07 & 40,85 & $-2,05$ \\
SC & 45,65 & 44,75 & 41,55 & 44,59 & 44,60 & $-1,05$ \\
RS & 44,30 & 41,51 & 35,01 & 38,49 & 39,65 & $-4,66$ \\
MS & 30,34 & 20,48 & 29,35 & 35,30 & 37,89 & 7,55 \\
MT & 39,32 & 34,00 & 41,33 & 33,55 & 37,26 & $-2,06$ \\
GO & 37,20 & 32,70 & 34,61 & 39,11 & 37,37 & 0,17 \\
DF & 50,46 & 52,59 & 49,76 & 53,87 & 50,53 & 0,07 \\
RO & 39,93 & 41,07 & 49,40 & 40,59 & 33,96 & $-5,97$ \\
AC & n.d. & 55,21 & 51,29 & 43,91 & 38,81 & $-16,40 *$ \\
AM & 44,25 & 42,44 & 39,96 & 46,67 & 41,80 & $-2,46$ \\
RR & 29,20 & n.d. & 51,72 & 56,33 & 48,45 & 19,24 \\
PA & 47,34 & 40,54 & 41,34 & 32,53 & 31,12 & $-16,23$ \\
AP & 76,25 & n.d. & 76,86 & 60,58 & 49,90 & $-26,34$ \\
TO & 36,75 & 21,74 & 23,66 & 29,36 & 31,27 & $-5,48$ \\
MA & 42,20 & 43,45 & 35,59 & 31,06 & 36,73 & $-5,47$ \\
PI & 46,85 & 36,16 & 44,47 & 43,77 & 39,77 & $-7,08$ \\
CE & 47,56 & 48,61 & 42,46 & 48,48 & 47,95 & 0,39 \\
RN & 48,57 & 47,68 & 39,13 & 50,61 & 52,01 & 3,44 \\
PB & 44,63 & 42,50 & 45,56 & 46,83 & 49,27 & 4,64 \\
PE & 51,39 & 43,20 & 37,96 & 45,33 & 43,42 & $-7,96$ \\
AL & 54,68 & 53,48 & 45,51 & 47,20 & 52,62 & $-2,05$ \\
SE & 42,09 & 45,51 & 49,60 & 41,13 & 40,57 & $-1,52$ \\
BA & 40,67 & 44,82 & 40,22 & 44,04 & 40,31 & $-0,36$ \\
\hline
\end{tabular}

Fonte: Elaboração própria a partir dos dados da PIA-IBGE (2016) 
Os dados assinalam que a densidade produtiva industrial do Brasil no ano de 2013 foi igual a 42,01\%, 4,70 p.p. inferior ao observado em 1996. Este resultado assinala que a indústria de transformação do país está perdendo capacidade de agregar valor nacionalmente.

Verifica-se que, em 19 dos 27 estados brasileiros, houve perda na relação VTI/VBPI, com destaque para o estado do Amapá, com perda relativa de 26,34 p.p. Por outro lado, oito estados obtiveram ganhos de densidade produtiva no período, sendo que os maiores aumentos foram verificados nos estados de Roraima (19,24 p.p.) e Mato Grosso do Sul (7,55 p.p.).

Na Região Sudeste, conhecida por ser a que apresenta os melhores índices industriais, todos os estados apresentaram uma queda relativa da relação VTI/VBPI, com destaque para o Rio de Janeiro (-6,94 p.p.), seguido de Espírito Santo (-6,72 p.p.), São Paulo (-5,78 p.p.) e Minas Gerais (-1,37 p.p.). Estes resultados sinalizam desindustrialização, pois mostra que estes estados estão utilizando maior volume de insumos importados ao longo do tempo. No caso do Rio de Janeiro e Espírito Santo, isso pode estar associado ao avanço das atividades de exploração de petróleo, o que requer a importação de máquinas e equipamentos para a atividade. Em São Paulo, apesar da grande exportação de bens industriais, o estado importa grande volume de matérias-primas e insumos industriais utilizados na produção.

Na Região Sul, todos os estados perderam densidade industrial, com o destaque para o Rio Grande do Sul (-4,66 p.p.), seguido de Paraná (-2,05 p.p.) e Santa Catarina (-1,05). No Centro Oeste, apenas o Mato Grosso $(-2,06)$ apresentou queda relativa da relação VTI/VBPI. Nas regiões Norte e Nordeste, quatro dos 16 estados apresentaram um aumento relativo da densidade produtiva industrial - Roraima (19,24 p.p.), Paraíba (4,64 p.p.), Rio Grande do Norte (3,44 p.p.) e Ceará (0,39 p.p.). Nos outros estados, foi verificado uma redução relativa de tal índice, com destaque para Amapá (-26,34 p.p.), Pará (-16,23 p.p.), Pernambuco (-7,96 p.p.) e Piauí (-7,08 p.p.).

Portanto, ao analisar os elos das cadeias industriais, com base no indicador de densidade produtiva, observa-se uma queda do mesmo ao longo do período 1996-2013, o que somado aos dois indicadores anteriores contribuem para identificar a ocorrência de desindustrialização.

A Tabela 5 apresenta a participação percentual do pessoal empregado na indústria de transformação de cada estado, demonstrando a capacidade da mesma em gerar emprego. $\mathrm{O}$ que se observa, é que, em relação ao Brasil, a industrial de transformação vem perdendo capacidade de gerar emprego em relação a indústria total (-0,64 p.p), fato este que ocorre na maioria dos estados brasileiros.

Pode-se perceber que 12 estados apresentaram um aumento relativo do número de pessoal ocupado na indústria de transformação, sendo a maioria destes localizados nas regiões Norte e Nordeste - Espírito Santo, Paraná, Goiás, Distrito Federal, Santa Catarina, Amazonas Ceara, Rio Grande do Norte, Paraíba, Pernambuco, Sergipe e Bahia. Por outro lado, 15 estados reduziram sua capacidade de gerar emprego, porém, apenas cinco deles - Goiás, Rio Grande do Norte, Amazonas, Sergipe e Bahia, tiveram aumento superior a 1 p.p, demonstrando que a indústria de transformação brasileira evoluiu pouco na geração de emprego.

Tabela 5: Participação do Pessoal Ocupado na Indústria de Transformação na Indústria Total, Unidades da Federação, 1996-2013 (em \%)

\begin{tabular}{lcccccc}
\hline & 1996 & 2000 & 2005 & 2010 & 2013 & $\Delta(2013-1996)$ \\
\hline BRASIL & $\mathbf{9 7 , 7 2}$ & $\mathbf{9 7 , 9 0}$ & $\mathbf{9 7 , 8 4}$ & $\mathbf{9 7 , 5 4}$ & $\mathbf{9 7 , 0 8}$ & $\mathbf{- 0 , 6 4}$
\end{tabular}




\begin{tabular}{ccccccc} 
MG & 93,68 & 94,91 & 94,91 & 94,16 & 93,61 & $-0,07$ \\
RJ & 96,63 & 95,72 & 93,23 & 91,56 & 91,11 & $-5,52$ \\
SP & 99,25 & 99,19 & 99,47 & 99,42 & 99,32 & 0,07 \\
ES & 98,42 & 98,78 & 99,09 & 99,19 & 99,10 & 0,68 \\
PR & 98,72 & 98,53 & 98,75 & 98,75 & 98,64 & $-0,08$ \\
SC & 99,06 & 99,32 & 99,36 & 99,21 & 99,22 & 0,16 \\
RS & 91,30 & 91,86 & 89,18 & 91,85 & 85,39 & $-5,91$ \\
MS & 97,41 & 98,31 & 97,92 & 97,59 & 97,04 & $-0,37$ \\
MT & 98,27 & 98,72 & 98,26 & 98,08 & 97,47 & $-0,80$ \\
GO & 96,85 & 97,03 & 97,35 & 97,82 & 97,97 & 1,12 \\
DF & 99,08 & 99,05 & 99,50 & 98,96 & 99,23 & 0,15 \\
RO & 95,63 & 98,40 & 98,37 & 96,56 & 95,56 & $-0,07$ \\
AC & n.d. & 100,00 & 99,73 & 98,87 & 99,36 & $-0,64 *$ \\
AM & 96,35 & 97,73 & 98,58 & 98,01 & 98,17 & 1,82 \\
RR & 100,00 & n.d. & 98,94 & 97,44 & 95,09 & $-4,91$ \\
PA & 91,97 & 96,10 & 94,31 & 89,27 & 80,53 & $-11,44$ \\
AP & 77,26 & n.d. & 93,97 & 80,09 & 73,43 & $-3,83$ \\
TO & 92,95 & 91,78 & 94,13 & 94,50 & 92,85 & $-0,10$ \\
MA & 98,77 & 97,73 & 94,86 & 95,41 & 86,89 & $-11,88$ \\
PI & 98,94 & 98,10 & 98,08 & 97,69 & 97,71 & $-1,23$ \\
CE & 98,76 & 98,87 & 99,16 & 98,94 & 98,79 & 0,03 \\
RN & 84,01 & 88,64 & 86,86 & 85,30 & 85,70 & 1,69 \\
PB & 97,31 & 96,82 & 97,47 & 98,13 & 98,01 & 0,70 \\
PE & 98,90 & 99,01 & 99,07 & 98,77 & 99,17 & 0,27 \\
AL & 99,51 & 99,06 & 99,26 & 99,41 & 99,32 & $-0,19$ \\
SE & 86,00 & 91,37 & 90,52 & 87,51 & 89,68 & 3,68 \\
BA & 91,09 & 93,07 & 94,27 & 94,75 & 93,80 & 2,71 \\
\hline
\end{tabular}

Fonte: Elaboração própria a partir dos dados da PIA-IBGE (2016) n.d.: dado não disponível

* variação entre 2000 e 2013

Na Região Sudeste, destaca-se a redução do emprego da indústria de transformação para o Rio de Janeiro (-5,52 p.p), o que pode ser explicado pelo ganho de força da indústria extrativa, puxada pelo petróleo. Em relação aos que aumentaram o pessoal ocupado na indústria de transformação, tem-se Espirito Santo (0,68 p.p) e São Paulo (0,07 p.p). Este último, apesar do baixo crescimento, tem mais de $99 \%$ do emprego industrial centrado na indústria de transformação. Cabe destacar que, Minas Gerais, embora tenha ampliado o emprego na indústria de transformação, tem uma participação expressiva de emprego gerado pela indústria extrativa, assim como o Rio de Janeiro.

A Região Sul reduziu a capacidade de gerar emprego na indústria de transformação, com exceção de Santa Catarina (0,16 p.p), demonstrando a possibilidade da existência de desindustrialização subnacional. O destaque fica para o Rio Grande do Sul (-5,91 p.p), em que houve um avanço significativo da participação no emprego industrial da indústria extrativa.

A Região Nordeste teve seis estados com aumento da capacidade da indústria de transformação de gerar emprego - Ceará, Rio Grande do Norte, Paraíba, Pernambuco, Sergipe e Bahia, porém apenas os dois últimos tiveram números mais expressivos. Por outro lado, o Maranhão (-11,88 p.p) foi o que mais perdeu emprego da indústria de transformação para a indústria extrativa.

Na Região Norte, grande parte dos estados perdeu participação de pessoal ocupado pela indústria de transformação, destacando o Pará (-11,44 p.p). O estado do Amazonas $(1,82 p . p)$ foi o único que apresentou aumento do indicador no período analisado.

Por último, na Região Centro Oeste, o emprego na indústria extrativa é pouco representativo, sendo que o estado de Goiás (1,12 p.p) e Distrito Federal (0,15 p.p) 
apresentaram aumento do pessoal ocupado na indústria de transformação. Por outro lado, Mato Grosso (-0,80 p.p) e Mato Grosso do Sul (-0,37 p.p) tiveram queda na capacidade da atividade de gerar emprego.

Assim, os dados da Tabela 5 corroboram com a ideia que existe um avanço de outros tipos de indústria em detrimento da indústria de transformação, como o caso da industrial extrativa. Esta ideia fica comprovada quando se observa o estado do Rio de Janeiro, a terceira queda mais intensa do país, onde a indústria extrativa ganhou muita força nos últimos anos.

A Tabela 6 apresenta a participação do emprego gerado pela indústria de transformação no emprego total de cada estado brasileiro. Neste caso, o objetivo é observar a capacidade da indústria de transformação de gerar emprego sobre o dinamismo da economia do estado, incluindo todos os outros setores não relacionados a indústria.

Na análise do dado para o país, percebe-se que a participação do emprego da indústria de transformação no total do emprego da economia reduziu-se em cerca de 4 p.p. entre 1996 e 2013.

Embora, em número absolutos, a evolução do emprego industrial ocorra na maioria dos estados brasileiros, as variações em termos relativos são bastante díspares, sendo que no período analisado, apenas oito das 27 Unidades da Federação aumentaram a participação do emprego da industrial de transformação em relação ao total. Entretanto, metade destes apresentaram uma variação superior a 2 p.p.

Tabela 6: Participação do Pessoal Ocupado na Indústria de Transformação no Emprego Total, Unidades da Federação, 1996-2013 (em \%)

\begin{tabular}{ccccccc}
\hline & 1996 & 2000 & 2005 & 2010 & 2013 & $\Delta(2013-1996)$ \\
\hline BRASIL & $\mathbf{2 0 , 7 4}$ & $\mathbf{1 9 , 5 3}$ & $\mathbf{1 8 , 7 0}$ & $\mathbf{1 7 , 5 2}$ & $\mathbf{1 6 , 7 4}$ & $-\mathbf{3 , 9 9}$ \\
MG & 19,15 & 18,54 & 17,81 & 17,04 & 16,77 & $-2,38$ \\
RJ & 14,15 & 12,30 & 10,65 & 10,16 & 9,88 & $-4,26$ \\
SP & 27,49 & 24,84 & 23,53 & 21,57 & 20,01 & $-7,48$ \\
ES & 14,66 & 15,60 & 13,71 & 13,31 & 12,52 & $-2,14$ \\
PR & 21,33 & 20,84 & 22,70 & 22,83 & 22,55 & 1,22 \\
SC & 36,93 & 34,66 & 33,66 & 31,91 & 30,89 & $-6,05$ \\
RS & 27,73 & 28,66 & 27,19 & 24,75 & 24,40 & $-3,33$ \\
MS & 9,74 & 11,20 & 11,83 & 13,40 & 14,01 & 4,27 \\
MT & 14,68 & 18,91 & 14,15 & 13,78 & 13,93 & $-0,75$ \\
GO & 14,63 & 14,69 & 14,64 & 15,93 & 16,69 & 2,06 \\
DF & 2,42 & 2,09 & 2,51 & 2,69 & 2,44 & 0,02 \\
RO & 11,53 & 12,76 & 10,97 & 8,09 & 9,94 & $-1,59$ \\
AC & .d. & 4,48 & 5,09 & 4,68 & 4,77 & 0,29 \\
AM & 25,41 & 23,43 & 23,68 & 19,97 & 21,08 & $-4,32$ \\
RR & 3,38 & $n . d$. & 4,42 & 2,32 & 2,25 & $-1,13$ \\
PA & 12,29 & 13,81 & 12,32 & 9,09 & 7,25 & $-5,04$ \\
AP & 4,41 & $n . d$. & 3,47 & 3,38 & 3,52 & $-0,89$ \\
TO & 3,56 & 5,29 & 5,07 & 5,42 & 6,12 & 2,56 \\
MA & 8,29 & 6,34 & 6,15 & 4,94 & 5,81 & $-2,48$ \\
PI & 8,02 & 8,12 & 7,46 & 6,59 & 6,79 & $-1,22$ \\
CE & 17,62 & 20,04 & 18,28 & 17,93 & 16,58 & $-1,04$ \\
RN & 11,50 & 13,38 & 11,16 & 11,88 & 9,94 & $-1,56$ \\
PB & 12,65 & 12,41 & 12,19 & 12,28 & 11,74 & $-0,90$ \\
PE & 15,19 & 14,47 & 14,64 & 13,97 & 13,53 & $-1,65$ \\
AL & 22,03 & 21,27 & 26,28 & 22,29 & 17,38 & $-4,66$ \\
SE & 8,87 & 10,72 & 10,01 & 9,79 & 11,14 & 2,27 \\
BA & 9,54 & 9,18 & 10,13 & 10,27 & 10,03 & 0,49 \\
\hline
\end{tabular}

Fonte: Elaboração própria a partir dos dados da PIA-IBGE (2016) e RAIS (2016). n.d.: dado não disponível. 
* variação entre 2000 e 2013.

Observa-se uma diminuição dos valores relativos do emprego industrial para todos os estados da Região Sudeste, sendo que os estados de São Paulo e Rio de Janeiro tiveram perdas acima da variação nacional. Assim, pode-se dizer que os dados de evolução do emprego se alinham aos de VTI, sugerindo que há um processo de desindustrialização relativa nesta região. Entretanto, observa-se uma desaceleração dos valores nos últimos anos analisados, não se mantendo a trajetória intensa de declínio.

Na Região Sul, o estado do Paraná (1,22 p.p) foi o único que apresentou aumento da participação relativa do emprego industrial. A contraposição destes dados com os de VTI evidencia as divergências entre os indicadores: enquanto para o VTI o estado de Santa Catarina apresentava resultado mais positivo, para o emprego industrial a melhor performance é a do Paraná, sendo que Rio Grande do Sul (-3,33 p.p) apresenta resultado negativo nos dois indicadores.

Na Região Nordeste, os estados de Sergipe (2,27 p.p) e Bahia (0,49 p.p) são os que vêm apresentando aumento da participação relativa do emprego industrial. Assim como verificado na região Sul, também no Nordeste há divergências entre os indicadores do PIB industrial e do emprego industrial.

Na Região Norte, dos sete estados, cinco apresentaram queda da participação relativa do emprego industrial, sendo Rondônia (-1,59 p.p), Amazonas (-4,32 p.p), Roraima (-1,13 p.p), Pará (-5,04 p.p) e Amapá (-0,89 p.p), resultado que também demonstra o pouco dinamismo da industrial neste estado

Por fim, na região Centro-Oeste, os estados que apresentaram aumento de valores relativos do emprego industrial foram Mato Grosso do Sul (4,27 p.p) e Goiás (2,06 p.p). No Distrito Federal, o indicador foi estável (0,02 p.p) e Mato Grosso contou com perda de participação (-0,75 p.p). No geral, os dados indicam que o aumento da participação do VTI industrial em relação ao PIB estadual foi acompanhado de crescimento da participação relativa do emprego industrial.

Cabe ressaltar que o decréscimo da participação do emprego industrial no emprego total pode resultar de aumentos de produtividade do setor, sem que essa redução seja explicada como perda de dinamismo da indústria (NASSIF, 2008). Deste modo, analisa-se, na sequência, a evolução do indicador de produtividade da indústria de transformação dos estados brasileiros, pela Tabela 7.

O indicador de produtividade foi calculado pela razão entre o Valor da Transformação Industrial (VTI) e pessoal ocupado (PO). A produtividade relaciona-se com a competitividade industrial, já que aquela pode ser compreendida como a capacidade empresarial de promover um aumento na produção com economia, sendo que ganhos de produtividade refletem em ganhos de rentabilidade e aumento da capacidade de fazer investimentos.

No que se refere ao Brasil, se observa um aumento de produtividade assim como em todas as unidades da federação. Porém, no caso do Brasil, a mesma aumentou em R \$ 81,24 mil/pessoa, variação menor do que apenas 8 estados, mostrando que grande parte dos estados aumentam a produtividade em nível inferior ao do país.

Tabela 7: Produtividade da Indústria de Transformação, Unidades da Federação, 19962013 (em R\$ mil/pessoa)

\begin{tabular}{ccccccc}
\hline & 1996 & 2000 & 2005 & 2010 & 2013 & $\Delta(2013-1996)$ \\
\hline BRASIL & $\mathbf{3 1 , 3 9}$ & $\mathbf{4 6 , 9 3}$ & $\mathbf{7 4 , 6 7}$ & $\mathbf{9 4 , 1 0}$ & $\mathbf{1 1 2 , 6 3}$ & $\mathbf{8 1 , 2 4}$ \\
MG & 27,65 & 40,88 & 69,55 & 92,71 & 102,73 & 75,08 \\
RJ & 32,57 & 56,83 & 107,69 & 139,22 & 155,08 & 122,51 \\
SP & 37,48 & 56,75 & 88,17 & 106,69 & 128,71 & 91,23
\end{tabular}




\begin{tabular}{ccccccc} 
ES & 27,03 & 42,90 & 67,30 & 88,38 & 110,16 & 83,13 \\
PR & 21,08 & 29,36 & 44,13 & 61,16 & 77,03 & 55,95 \\
SC & 25,30 & 38,40 & 57,51 & 80,37 & 101,56 & 76,27 \\
RS & 26,93 & 54,05 & 83,74 & 80,66 & 105,10 & 78,17 \\
MS & 21,97 & 23,35 & 53,91 & 82,45 & 124,19 & 102,22 \\
MT & 22,14 & 21,85 & 86,80 & 103,43 & 136,21 & 114,07 \\
GO & 21,95 & 26,38 & 55,36 & 81,22 & 100,21 & 78,25 \\
DF & 23,15 & 37,64 & 52,67 & 70,98 & 82,03 & 58,88 \\
RO & 8,17 & 12,74 & 44,53 & 67,79 & 76,37 & 68,20 \\
AC & n.d. & 11,76 & 22,68 & 44,02 & 53,21 & $41,45 *$ \\
AM & 89,92 & 133,17 & 187,43 & 242,00 & 238,31 & 148,39 \\
RR & 5,25 & n.d. & 22,83 & 33,68 & 37,24 & 31,99 \\
PA & 22,30 & 31,32 & 53,14 & 60,37 & 71,11 & 48,81 \\
AP & 29,22 & n.d. & 97,38 & 33,00 & 112,58 & 83,36 \\
TO & 14,07 & 15,02 & 30,66 & 46,42 & 68,17 & 54,10 \\
MA & 24,60 & 48,65 & 66,89 & 57,70 & 87,15 & 62,55 \\
PI & 12,04 & 11,92 & 33,00 & 60,74 & 50,77 & 38,73 \\
CE & 16,79 & 23,89 & 31,69 & 43,77 & 56,85 & 40,05 \\
RN & 12,03 & 16,72 & 23,96 & 45,21 & 68,63 & 56,60 \\
PB & 14,40 & 19,93 & 34,13 & 44,23 & 57,64 & 43,24 \\
PE & 20,32 & 22,69 & 33,81 & 56,23 & 70,19 & 49,87 \\
AL & 17,28 & 23,33 & 21,83 & 26,77 & 42,24 & 24,97 \\
SE & 17,28 & 29,05 & 50,42 & 53,38 & 60,63 & 43,36 \\
BA & 43,45 & 89,64 & 138,30 & 146,93 & 159,96 & 116,51 \\
\hline
\end{tabular}

Fonte: Elaboração própria a partir dos dados da PIA-IBGE (2016). n.d.: dado não disponível.

* variação entre 2000 e 2013.

Na região Sudeste, os estados de São Paulo, Rio de Janeiro e Espírito Santo apresentaram crescimento da produtividade superior à média brasileira. Por outro lado, Minas Gerais, apesar do crescimento da produtividade no período, ficou aquém do indicador nacional. Nas regiões Sul, Nordeste e Centro-Oeste, o crescimento da produtividade foi menor do que a média brasileira para a grande maioria de seus estados. As únicas exceções são os estados da Bahia, na região Nordeste, e os estados de Mato Grosso e Mato Grosso do Sul, na região Centro-Oeste, que apresentaram elevação da produtividade superior à média brasileira.

Deste modo, no geral, apenas a região Sudeste contou com aumento do indicador de produtividade maior do que o dado brasileiro. Como nesta região houve perda de participação dos empregos industriais no emprego total, há um importante indício de que esta perda se associa, em alguma medida, ao aumento da produtividade industrial. Sendo assim, este decréscimo da participação do emprego industrial não evidencia desindustrialização.

Em síntese, em todas as regiões foi observado ganhos de produtividade ao longo do período analisado, trazendo a ideia da possibilidade de avanços tecnológicos observado no período de 1996 a 2013. Assim, ao analisar somente o pessoal ocupado pela indústria de transformação fica vaga a ideia de desindustrialização, pois uma redução de pessoal pode ser advinda de ganhos de produtividade, em que se consegue gerar mais valor por unidade de mão de obra empregada.

Na Tabela 8, a seguir, verifica-se a composição das exportações estaduais em termos da participação dos produtos básicos, semimanufaturados e manufaturados. Este indicador permite analisar o perfil exportador de cada estado, e será possível observar o aumento da participação dos produtos básicos no período de 2000 a 2015. 
Os dados indicam que 21 das 27 Unidades da Federação apresentaram aumento da participação das exportações de produtos básicos na pauta. Contudo, isto não significa que a composição no ano de 2015 é especializada neste tipo de produto, como o caso dos estados de Santa Catarina, Maranhão, Sergipe e Bahia. Por outro lado, quando se analisa os seis estados em que as exportações de produtos básicos caíram ao longo do período analisado, dois deles, Mato Grosso do Sul e Goiás são fortemente especializados em produtos básicos.

Na Região Sul e Sudeste, o que se observa é que todos os estados analisados apresentam aumento das exportações dos produtos básicos, destaque para o estado do Rio de Janeiro, aonde a variação de 2000 a 2010 foi aproximadamente de $460 \%$, explicado por conta do aumento das exportações de óleo bruto de petróleo, no qual o estado do Rio é mais representativo do que todos os demais.

Nas Regiões Norte e Nordeste, apenas quatro dos 16 estados reduziram o peso das exportações de produtos básicos, demonstrando que na maioria dos estados destas regiões o que se observa é um reprimarização da pauta exportadora. Como citado anteriormente, apesar de aumentar a exportação de básicos, os estados do Maranhão, Sergipe e Bahia, no ano de 2015, ainda apresentam sua pauta exportadora especializada em produtos manufaturados, sendo que no Sergipe aproximadamente $98 \%$ das exportações são deste tipo de produto.

Tabela 8: Composição das Exportações Estaduais, Unidades da Federação, 2000-2015 (em \%)*

\begin{tabular}{ccccc|ccc}
\hline \multicolumn{7}{c}{$\mathbf{2 0 0 0}$} & \multicolumn{3}{c}{$\mathbf{2 0 1 5}$} \\
\hline BRASIL & Básicos & $\mathbf{2 2 , 7 9}$ & $\mathbf{S e m i .}$ & Manufaturados & Básicos & Semi. & Manufaturados \\
MG & 42,80 & 24,44 & $\mathbf{5 9 , 0 7}$ & $\mathbf{4 5 , 6 2}$ & $\mathbf{1 3 , 8 5}$ & $\mathbf{3 8 , 0 8}$ \\
RJ & 9,52 & 7,39 & 70,73 & 55,50 & 23,39 & 21,01 \\
SP & 5,23 & 5,71 & 87,11 & 53,19 & 7,78 & 35,22 \\
ES & 33,79 & 55,78 & 7,88 & 13,35 & 11,85 & 71,19 \\
PR & 37,81 & 11,35 & 49,12 & 56,03 & 22,20 & 21,11 \\
SC & 25,61 & 4,93 & 69,43 & 44,17 & 11,11 & 36,41 \\
RS & 26,73 & 10,79 & 61,34 & 53,16 & 3,48 & 52,30 \\
MS & 80,61 & 7,68 & 11,68 & 65,38 & 31,83 & 38,17 \\
MT & 84,29 & 9,05 & 6,52 & 95,43 & 3,55 & 2,79 \\
GO & 74,16 & 21,08 & 4,76 & 72,85 & 22,05 & 5,02 \\
DF & 6,02 & - & 65,84 & 80,53 & 0,59 & 6,09 \\
RO & 2,73 & 72,92 & 21,38 & 77,59 & 7,89 & 11,14 \\
AC & 6,57 & 64,43 & 27,30 & 72,10 & 3,81 & 24,09 \\
AM & 1,32 & 0,53 & 97,12 & 4,27 & 2,31 & 90,86 \\
RR & 2,73 & 72,92 & 21,38 & 77,59 & 7,89 & 11,14 \\
PA & 44,51 & 41,11 & 14,36 & 70,61 & 11,57 & 17,59 \\
AP & 32,62 & 59,70 & 7,69 & 3,61 & 83,42 & 12,95 \\
TO & 55,11 & 44,89 & - & 98,35 & 1,49 & 0,15 \\
MA & 12,00 & 77,54 & 10,38 & 29,51 & 34,68 & 35,00 \\
PI & 30,68 & 44,32 & 24,98 & 85,80 & 13,40 & 0,80 \\
CE & 40,19 & 16,64 & 40,97 & 27,01 & 22,75 & 48,47 \\
RN & 54,75 & 4,23 & 40,39 & 57,49 & 0,86 & 39,74 \\
PB & 20,57 & 4,96 & 74,43 & 14,02 & 7,00 & 78,98 \\
PE & 17,09 & 27,77 & 51,35 & 13,26 & 7,88 & 76,09 \\
AL & 2,40 & 74,66 & 22,93 & 1,10 & 64,39 & 34,47 \\
SE & 0,16 & 4,51 & 95,21 & 1,26 & 0,55 & 98,19 \\
BA & 14,24 & 25,99 & 57,23 & 29,65 & 32,10 & 37,27 \\
\hline
\end{tabular}

Fonte: Elaboração própria a partir dos dados do MDIC (2016).

* Exclui operações especiais. 
Por fim, na Região Centro-Oeste, que se destacou na evolução da maioria dos índices industriais, o que se observa é que dois estados - Mato Grosso do Sul e Goiás - perderam participação dos produtos básicos nas exportações, porém, no ano de 2015, as exportações dos produtos básicos ainda são a principal fonte exportadora da região, representando mais de $65 \%$ das exportações em todos os estados que a compõem.

O Quadro 1 sintetiza os resultados encontrados pela pesquisa realizada para a elaboração deste trabalho. Em termos gerais, as evidências preliminares fornecidas pelos indicadores industriais, e, tomando como referência o conceito de desindustrialização relativa, verifica-se que para a maior parte dos estados brasileiros há evidências de um processo de desindustrialização em curso.

Quadro 1: Síntese dos Indicadores

\begin{tabular}{|c|c|c|c|c|c|c|c|}
\hline Estados & $\begin{array}{c}\text { VTI } \\
\text { Estado/ } \\
\text { Brasil }\end{array}$ & $\begin{array}{c}\text { Densidad } \\
\text { e }\end{array}$ & PO* & $\begin{array}{c}\text { Produtivid } \\
\text { ade }\end{array}$ & $\begin{array}{c}\text { VTI/P } \\
\text { IB }\end{array}$ & $\begin{array}{c}\text { Exportações } \\
\text { industriais }\end{array}$ & Resultado \\
\hline MG & 1 & - & - & + & + & - & - \\
\hline $\mathbf{R J}$ & 1 & - & - & + & I & - & - \\
\hline SP & - & - & - & + & - & + & - \\
\hline ES & 1 & - & - & + & 1 & - & - \\
\hline PR & + & - & + & + & + & - & + \\
\hline SC & + & - & - & + & + & + & + \\
\hline RS & 1 & - & - & + & + & - & - \\
\hline MS & 1 & + & + & + & + & - & + \\
\hline MT & + & - & / & + & + & - & + \\
\hline GO & + & 1 & + & + & + & - & + \\
\hline DF & 1 & 1 & 1 & + & 1 & - & - \\
\hline RO & 1 & - & - & + & + & - & - \\
\hline AC & I & - & I & + & + & - & - \\
\hline AM & 1 & - & - & + & + & + & + \\
\hline RR & 1 & + & - & + & 1 & - & - \\
\hline PA & 1 & - & - & + & - & - & - \\
\hline AP & / & - & / & + & - & + & - \\
\hline TO & 1 & - & + & + & + & - & + \\
\hline MA & 1 & - & - & + & 1 & + & - \\
\hline PI & 1 & - & - & + & 1 & - & - \\
\hline $\mathbf{C E}$ & 1 & 1 & - & + & + & + & + \\
\hline RN & 1 & + & - & + & + & - & + \\
\hline PB & 1 & + & 1 & + & + & + & + \\
\hline PE & 1 & - & - & + & 1 & + & - \\
\hline AL & I & - & - & + & - & + & - \\
\hline SE & 1 & - & + & + & + & + & + \\
\hline BA & + & 1 & 1 & + & + & + & + \\
\hline
\end{tabular}

Fonte: Elaboração própria conforme Botelho et al. (2014).

*: Pessoal ocupado indústria de transformação / pessoal ocupado total.

Nota: A partir do valor da variação percentual de cada indicador entre 1996 e 2013, adotou-se como critério de classificação: variação positiva acima de 1\% foi atribuída o símbolo de mais (+), representando inexistência de tendência de desindustrialização relativa; variação entre -1\% e 1\%, foi considerada estável, sendo simbolizada pela barra (/); variação negativa, com uma redução superior a $-1 \%$, significa tendência a desindustrialização relativa, representada pelo sinal de menos (-).

O fato de os principais indicadores positivos (não ocorrência de desindustrialização) situarem-se nos estados da região Centro-Oeste e no Nordeste do país deve ser analisado com cautela, em função de serem regiões cuja base industrial é proporcionalmente pequena em relação aos estados do Sudeste e do Sul. Nestes casos, em especial no Centro Oeste, os 
resultados positivos parecem estar relacionados mais intensamente à agroindústria, decorrente da expansão da fronteira agrícola nestas regiões. Além disso, cabe destacar que, especialmente na região Sudeste, verifica-se um avanço significativo da indústria extrativa em Minas Gerais, Espírito Santo e Rio de Janeiro, o que também colabora para a obtenção de menores resultados para a indústria de transformação da região.

\section{CONCLUSÕES}

A desindustrialização tem estado no centro dos recorrentes debates acerca dos reduzidos níveis de expansão da atividade econômica do Brasil anos recentes. Seguindo essa problemática, esse trabalho dedicou-se a uma análise regionalizada da indústria, a fim de verificar se ocorre uma incidência desigual do processo, com sinais mais evidentes nas regiões mais industrializadas do país.

A elaboração de um conjunto de indicadores sobre o desempenho da indústria de transformação possibilitou extrair informações sobre a evolução industrial das regiões/estados quanto à agregação de renda, produtividade, densidade industrial, emprego e participação na atividade econômica local e nacional, os quais se constituem como base para aferições acerca da ocorrência ou não de um processo de desindustrialização na economia brasileira.

Deste modo, os dados analisados neste trabalho oferecem indícios preliminares em linha com a tese de um processo de desindustrialização em curso na economia brasileira, visto que os dados relativos sugerem perda de importância da indústria como motor da economia.

Pode-se concluir que a região Sudeste é mais afetada pelo processo de desindustrialização, pois todos os estados desta região perderam participação relativa da indústria de transformação como fator de dinamismo na economia. Por outro lado, os ganhos da indústria de transformação se concentraram nas regiões Centro-Oeste e Nordeste. Este fato se alinha à ideia que foi discutida ao longo do texto de que o processo de desindustrialização se dá de forma desigual na economia, além do que está ocorrendo alguma desconcentração da atividade industrial do Sul e Sudeste para o Centro Oeste e Nordeste.

Outro aspecto importante identificado pelos indicadores trabalhados neste artigo é que, quando da recuperação do crescimento econômico a partir de 2009 as reações dos estados em termos de crescimento industrial são divergentes, sendo que alguns apresentam inflexão na trajetória de crescimento anterior, e outros apresentam uma recuperação do crescimento industrial, no caso deste trabalho, em 2010 e 2013.

Entretanto, apesar das influências das políticas econômicas dos anos 1990, o que se observa é que a ausência de uma política industrial "agressiva” faz com que o parque industrial brasileiro fique defasado tecnologicamente, tornando este processo de desindustrialização ainda mais intenso.

Por fim, para o segundo momento desta pesquisa, serão estimados modelos econométricos por estados considerando os efeitos de algumas variáveis apontadas pela literatura como relevantes para explicar o processo de desindustrialização, tais como a taxa de câmbio real, taxa de juros, abertura comercial, preços de commodities, para tentar captar os impactos das mesmas sobre a atividade industrial.

\section{REFERÊNCIAS}

ALMEIDA, T. R. C.; SOUZA, C. C. A. Evolução da estrutura industrial de Minas Gerais no período 1960-2010: uma análise frente aos demais estados da federação. Anais do XVI Seminário sobre a Economia Mineira em Diamantina, Diamantina, 2014. 
ARROYO, M. Circuitos espaciais de produção industrial e fluxos internacionais de mercadorias na dinâmica territorial do estado de São Paulo. Boletim Campineiro de Geografia. v. 2, n. 1, 2012.

BARBOSA, W.; CARMO, A. S. S.; RAIHER, A. P. Existe desindustrialização no Estado do Paraná? um teste empírico para o período de 1996 a 2012. Informe Gepec. Toledo, v.19, n.i, p.55-79, jan./jun. 2015.

BOTELHO, M. dos R. A.; SOUSA, G. de F.; AVELLAR, A. P. M. de. A incidência desigual do processo de desindustrialização nos estados brasileiros. Anais do XVI Seminário sobre a Economia Mineira de Diamantina, Diamantina, 2014.

BRESSER-PEREIRA, L. C.; MARCONI, N. Existe Doença Holandesa no Brasil? IV Fórum de Economia da Fundação Getúlio Vargas. Março, 2008. Disponível em: http://www.bresserpereira.org.br. Acesso em: 20/11/2015.

BRUNO, M., ARAÚJO, E. \& PIMENTEL, D. Regime Cambial e Mudança Estrutural na Indústria de Transformação Brasileira: Novas Evidências para o Período (1994-2008). Anais do XXXVII Encontro Nacional de Economia ANPEC, Foz do Iguaçu, Dezembro, 2009.

CANO, W. A desindustrialização no Brasil. Economia e Sociedade, v. 21, número especial, p. 831-851, Campinas. 2012.

CARVALHO, L.; KUPFER, D. A transição estrutural da indústria brasileira: da diversificação para a especialização. Anais do XXXV Encontro Nacional de Economia da ANPEC, Recife. 2007.

CAVALIERI, H.; CARIO, S. A. F.; FERNANDES, R. L. Estrutura industrial brasileira e de Santa Catarina: alguns indícios de desindustrialização. Indic. Econ. FEE, v. 40, n. 3, p. 81104, Porto Alegre. 2013.

FERREIRA, J. S. W. São Paulo: o Mito da Cidade-Global. 2003. 336f. Tese (Doutorado). Faculdade de Arquitetura e Urbanismo, Universidade de São Paulo, São Paulo, 2003.

IBGE. Instituto Brasileiro de Geografia e Estatística. Indicadores. Contas Nacionais. Disponível em: http://www.ibge.gov.br/home/estatistica/indicadores/pib/defaultcnt.shtm. Acesso em Abr. 2016.

KALDOR, N. Causes of the slow rate of economic growth of the United Kingdom: an inaugural lecture. Cambridge University Press, 1966.

LIBÂNIO, G. Pauta de Exportações e Crescimento Econômico no Nordeste. In: Anais do XVII Encontro Regional de Economia. Fórum BNB de Desenvolvimento. 2012. Disponível em:

https://www.bnb.gov.br/content/aplicacao/eventos/forumbnb2012/docs/sim1_mesa1_pauta_e xportacoes_crescimento_economico_nordeste.pdf. Acesso em: 20/11/2015.

VERÍSSIMO, M. P. Doença Holandesa no Brasil: Ensaios sobre Taxa de Câmbio, Perfil Exportador, Desindustrialização e Crescimento Econômico. 2010. Tese (Doutorado em 
Economia) - Instituto de Economia da Universidade Federal de Uberlândia. Uberlândia/MG. 2010.

MDIC. Ministério do Desenvolvimento, Indústria e Comércio Exterior. Estatísticas de Comércio Exterior (DEPLA). Disponível em: http://www.desenvolvimento.gov.br/sitio/interna/interna.php?area=5\&menu=1161. Acesso em Abr. 2016.

MEDEIROS, R. L.; LANNES JUNIOR, M. A. O desafio da inovação industrial no Espírito Santo. Anais do X Congresso Nacional de Excelência em Gestão. Rio de Janeiro, Agosto. 2014.

MONTEIRO, F. D. S. C.; LIMA, J. P. R. Desindustrialização regional no Brasil. Anais do III Encontro Pernambucano de Economia. Recife, Novembro, 2014.

OREIRO, J. L.; FEIJÓ, C. A. Desindustrialização: Conceituação, Causas, Efeitos e o Caso Brasileiro. Revista de Economia Política, Vol. 30, nº. 2, Abr-Jun, 2010.

PALMA, J. G. Quatro fontes de desindustrialização e um novo conceito de doença holandesa. Federação das Indústrias do Estado de São Paulo. São Paulo. 2005.

PNUD. Programa das Nações Unidas para o Desenvolvimento. IDHM UF. Disponível em: http://www.pnud.org.br/atlas/ranking/Ranking-IDHM-UF-2010.aspx. Acesso em: Abr. 2016.

REZENDE, F. A. E.; SANTOS, F. Desindustrialização, rearranjo industrial e desemprego no Brasil. O caso do ABC paulista. Revista Eletrônica, ano 1, n. 1, p. 31-34. 2007.

RAIS. Relação Anual de Informações. Ministério do Trabalho e da Previdência Social. Disponível em: http://www.rais.gov.br/sitio/index.jsf. Acesso em: Abril. 2016.

ROSENDO, R. C.; BRITTO, J. Evolução da Densidade Industrial do Estado do Rio de Janeiro: Análise Comparativa com os Estados do Sudeste Brasileiro - 2000/2005. Anais do 39. Encontro Nacional de Economia. Foz do Iguaçu. Dezembro. 2011.

ROWTHORN, R.; WELLS, J. R. De-Industrialization and Foreign Trade. Cambridge, Cambridge University Press. 1987.

TEIXEIRA, F. W.; RODOLFO, F. Trajetória recente do setor industrial catarinense: uma análise com base nos dados da produção física. Revista NECAT, ano 1, n. 1, p. 22-30, Florianópolis. 2012. 